\title{
Computed Tomography Reconstruction Checklist for Data Acquired on the ISA MicroCT System
}

\author{
William D. Brown \\ Lawrence Livermore National Laboratory \\ Livermore, CA 94551 \\ Work performed for the \\ Science \& Technology Directorate of the \\ Department of Homeland Security \\ Statement of Work \\ PR RSEN-08-00066 \\ January 23, 2014 \\ Version 1.1 \\ LLNL-TR-649121 \\ IM\# 768325
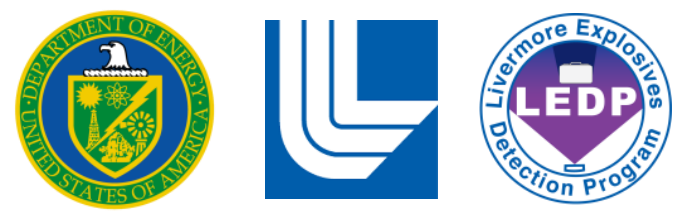
This document was prepared as an account of work sponsored by an agency of the United States government. Neither the United States government nor Lawrence Livermore National Security, LLC, nor any of their employees makes any warranty, expressed or implied, or assumes any legal liability or responsibility for the accuracy, completeness, or usefulness of any information, apparatus, product, or process disclosed, or represents that its use would not infringe privately owned rights. Reference herein to any specific commercial product, process, or service by trade name, trademark, manufacturer, or otherwise does not necessarily constitute or imply its endorsement, recommendation, or favoring by the United States government or Lawrence Livermore National Security, LLC. The views and opinions of authors expressed herein do not necessarily state or reflect those of the United States government or Lawrence Livermore National Security, LLC, and shall not be used for advertising or product endorsement purposes.

This work performed under the auspices of the U.S. Department of Energy by Lawrence Livermore National Laboratory under Contract DE-AC52-07NA27344. 


\section{Computed Tomography Reconstruction Checklist for Data Acquired on the ISA MicroCT System}

\subsection{Purpose}

This procedure describes the steps to reconstruct $\mathrm{X}$-ray projection images acquired from the ISA MicroCT system using the Lawrence Livermore National Laboratory (LLNL) developed software (IMGREC).

\subsection{Special Tools, Equipment, and Software}

1) A computer running Microsoft Windows ${ }^{\circledR}$

2) IMGREC software version 2.8.1.1c11 or later

3) Read and write access to the reconstruction computer

4) Portable hard drive to copy data from the data acquisition computer to the reconstruction computer

5) MicroCT beam hardening correction file (YYMMDD_ISA_BHC_Coeffs.txt) where:

YY is the year the beam correction data file was generated

MM is the month the beam correction data file was generated

$\mathrm{DD}$ is the day the beam correction data file was generated

6) MicroCT reconstruction scripts (YYMMDD_Script_Exp1.txt, YYMMDD_Script_Exp2.txt, YYMMDD_Script_Exp1_Open.txt) where:

$\mathrm{YY}$ is the year the beam correction data file was generated

$\mathrm{MM}$ is the month the beam correction data file was generated

$\mathrm{DD}$ is the day the beam correction data file was generated

\subsection{Setup Reconstruction Folders}

Create a new directory on the reconstruction computer titled

'YYMMDD_Sample' where:

YY is the year the beam correction data file was generated

$\mathrm{MM}$ is the month the beam correction data file was generated

DD is the day the beam correction data file was generated

Sample is a unique identifier for the sample acquired

Inside the new directory create 3 additional subdirectories titled 'Exp1', Exp2', and 'Exp1_Open'. Note: The 'E' in Exp1, Exp2 and Exp1_Open must be capitalized.

Place the projection data acquired for Exp1 into the directory titled 'YYMMDD_SamplelExp1'.

Place the projection data acquired for Exp2 into the directory titled 'YYMMDD_SamplelExp2'.

Place the projection data acquired for Exp1_Open into the directory titled 'YYMMDD_SamplelExp1_Open'. 


\subsection{Reconstruct Exp1 Data}

Place the reconstruction script 'YYMMDD_Script_Exp1.txt' into the directory 'YYMMDD_SamplelExp1'.

Double click on the IMGREC icon to start the IMGREC software

A new window will open requesting the user to set current directory. Navigate to directory 'YYMMDD_SamplelExp1' and click 'done'.

Click on 'FileIO'. Inside the 'FileIO" pull down menu select 'ReadComFile'.

A new window will open. Select 'YYMMDD_Script_Exp1.txt' and click 'Load'.

A new 'Command File Processing' window will load. Click 'Edit-Com' and this will open the script file. Change line 5 of the script to read 'rdsct Filename' where Filename is the name of the projection images. Click 'File/Save' and close the script.

In the 'Command File Processing' window click 'Do-Com-File'.

The projection data will be processed and $\mathrm{CT}$ reconstruction data will be generated. When the 'Current Command' inside the 'Command File Processing' states 'Reconstruction Complete', the reconstruction process is complete.

Click on 'Quit' to quit and exit the IMGREC software

\subsection{Reconstruct Exp2 Data}

Place the reconstruction script 'YYMMDD_Script_Exp.txt' into the directory 'YYMMDD_SamplelExp2'.

Place the Beam Hardening Correction file ('YYMMDD_ISA_BHC_Coeffs.txt ') into directory ' $\mathrm{C}$ : ct lbmcorfiles'.

Double click on the IMGREC icon to start the IMGREC software

A new window will open requesting the user to set current directory. Navigate to directory 'YYMMDD_SamplelExp2' and click 'done'.

Click on 'FileIO'. Inside the 'FileIO" pull down menu select 'ReadComFile'.

A new window will open. Select 'YYMMDD_Script_Exp2.txt' and click 'Load'. A new 'Command File Processing' window will load. Click 'Edit-Com' and this will open the script file. Change line 5 of the script to read 'rdsct Filename' where Filename is the name of the projection images. Click 'File/Save' and close the script.

In the 'Command File Processing' window click 'Do-Com-File'.

The projection data will be processed and $\mathrm{CT}$ reconstruction data will be generated. When the 'Current Command' inside the 'Command File Processing' states 'Reconstruction Complete', the reconstruction process is complete.

Click on 'Quit' to quit and exit the IMGREC software

\subsection{Reconstruct Exp1_Open Data}

Place the reconstruction script 'YYMMDD_Script_Exp1_Open.txt' into the directory 'YYMMDD_SamplelExp1_Open'.

Double click on the IMGREC icon to start the IMGREC software

A new window will open requesting the user to set current directory. Navigate to directory 'YYMMDD_SamplelExp1_Open' and click 'done'.

Click on 'FileIO'. Inside the 'FileIO" pull down menu select 'ReadComFile'. 
$\square$ A new window will open. Select 'YYMMDD_Script_Exp1_Open.txt' and click 'Load'.

$\square$ A new 'Command File Processing' window will load. Click 'Edit-Com' and this will open the script file. Change line 5 of the script to read 'rdsct Filename' where Filename is the name of the projection images. Click 'File/Save' and close the script.

$\square \quad$ In the 'Command File Processing' window click 'Do-Com-File'.

$\square \quad$ The projection data will be processed and CT reconstruction data will be generated. When the 'Current Command' inside the 'Command File Processing' states 'Reconstruction Complete', the reconstruction process is complete.

$\square$ Click on 'Quit' to quit and exit the IMGREC software 